\title{
L'âme et les sensations selon Charles Bonnet \\ (1720-1793)
}

par Patrick Baud

\section{RÉSuMÉ}

Bonnet a apporté à l'histoire naturelle d'importantes contributions, comme expérimentateur et comme théoricien. Des troubles de la vue le contraignent à renoncer, jeune encore, à l'observation; il s'oriente alors vers la psychologie et la philosophie, organisant sa réflexion autour de deux thèmes majeurs : l'origine de nos idées et l'organisation de nos facultés.

Le nom de Charles Bonnet, né à Genève en 1720, est associé dans l'histoire de la biologie à la découverte de la parthénogenèse du puceron. Cette importante observation lui valut immédiatement l'estime du monde savant: âgé de vingt ans à peine, il devint en 1740 correspondant de l'Académie des Sciences, grâce à l'appui de Réaumur.

Les contributions de Bonnet à l'histoire naturelle sont nombreuses et diverses. Il s'est illustré aussi bien par ses recherches expérimentales sur les insectes, la régénération animale (chez les vers, les polypes, la salamandre, etc.), l'anatomie et la physiologie végétale (travaux sur les fonctions des feuilles, la circulation de la sève, le tropisme des racines, etc.), que par sa réflexion théorique. Dans ses ouvrages les plus célèbres (Considérations sur les corps organisés, 1762; Contemplation de la Nature, 1765), il aborde les questions de l'origine et de la génération des êtres vivants et se révèle un brillant défenseur des théories préformationnistes.

Ce sont les circonstances plus que des inclinations précoces qui vont orienter Bonnet, naturaliste heureux, vers la psychologie et la philosophie. Il est encore assez jeune lorsque des troubles de la vue le contraignent à renoncer aux longues heures d'observation. Ménageant ses yeux, il se livre alors à la méditation et se consacre à des ouvrages de philosophie et de psychologie, «flatté de se produire au monde comme métaphysicien» ${ }^{1}$. Pour se diriger dans ces matières nouvelles, il sollicite les méthodes d'observation et d'analyse qui ont fait leurs preuves dans l'étude de la nature: «J'ai entrepris d'étudier l'homme comme j'ai étudié les insectes et les plantes» ${ }^{2}$. 
La contribution de Bonnet à la psychologie mérite d'être soulignée, pour la vigueur, l’originalité et parfois même l'intrépidité de ses vues («... je ne me suis pas effrayé des conséquences» déclare-t-il dans la préface à l'Essai de Psychologie).

Son projet est explicitement formulé dans la préface à l'Essai analytique sur les facultés de l'âme: c'est à l'étude de notre être que Bonnet entend se consacrer, et particulièrement à l'examen de «la mécanique de nos idées», en les considérant «dans l'instrument qui sert à leur formation, à leur rappel et à leur enchaînement». Aussi accorde-t-il toute son attention à l'organe des sensations - au cerveau, aux nerfs - et surtout à la fibre, élément matériel ultime sur lequel il fonde son argumentation et ses démonstrations. «Je bâtis toujours sur le physique de notre être, je ne vais à l'âme que par le corps», répétera-t-il beaucoup plus tard (MA, p. 174). Là réside le caractère novateur de sa psychologie, mais aussi, notons-le d'emblée, la source de l'accusation injustifiée - de matérialisme dont il eut parfois à se défendre. Par ce choix méthodologique, hérité de sa formation de naturaliste, par l'utilisation conjointe de l'analyse et de l'introspection, il dépouille de ses attributs métaphysiques la psychologie de son temps et annonce la psychologie scientifique, et à certains égards la psychophysiologie. «Je souhaiterais que ce petit ouvrage fût une psychologie expérimentale et géométrique», écrit-il de l'Essai analytique (EA, 56).

Les principes fondamentaux de la psychologie de Bonnet sont exposés en détail dans l'Essai de Psychologie (EP, 1755) ${ }^{3}$ et dans l'Essai analytique sur les facultés de l'âme (EA, 1760) ${ }^{4}$. Il en présentera encore une «légère esquisse» dans la préface à la Contemplation de la Nature, reprise sous la forme d'une «Analyse abrégée de l'Essai analytique» dans La Palingénésie philosophique $(\mathrm{PP}, 1770)^{5}$, qui résume et approfondit les grands thèmes de son œuvre.

Bonnet adhère au dualisme cartésien: l'homme est un être mixte, composé de deux substances, l'âme et le corps. Principe de nos facultés, l'âme est une, simple et immatérielle. Si l'union de l'âme et du corps est pour lui un fait certain, Bonnet ne se prononce ni sur le lieu ni sur la nature de cette union. Ses conceptions à cet égard trahissent d'ailleurs une indécision significative, nous semble-t-il, de l'évolution des idées à son époque, et du «conflit» en lui de l'héritage cartésien et de l'influence sensualiste. Car Bonnet fait sienne la conception de Locke selon laquelle toutes nos idées nous viennent des sens. Cette vue s'impose alors si nettement que Bonnet peut même écrire: 
«Tous les philosophes conviennent aujourd'hui que nos idées tirent leur origine des sens» (EA, préface).

Ou encore:

«Je suis parti d'un fait très connu, très certain, et que personne ne s'avisera jamais de contester: c'est qu'un aveugle-né n'acquerra jamais nos idées de lumière et de couleur» (PP, p.3).

L'Essai de psychologie et l'Essai analytique sont consacrés aux mêmes thèmes - l'origine de nos idées, l'organisation de nos facultés - mais d'un ouvrage à l'autre Bonnet renouvelle les principes d'exposition et de démonstration. Dans le premier, il suit le développement psychologique et «cognitif» d'un individu, de la vie fœtale à l'âge adulte, et montre comment les sensations donnent naissance aux idées et à la vie intellectuelle. Il s'agit, en quelque sorte, d'une ontogenèse des facultés, d'une psychogenèse. Dans l'Essai analytique, il imagine une statue qui possèderait tous les organes et tous les attributs physiques d'un adulte sain: vierge de toute sensation, elle s'animera progressivement au gré des fictions du philosophe. Bonnet en a déjà commencé la rédaction lorsque paraît en 1754 le Traité des Sensations de Condillac. Devant la convergence de leurs démarches, il envisage tout d'abord de renoncer à son projet, puis se laisse convaincre par un ami qui l'incite à poursuivre.

«Vous ferez, lui dit-il, un ouvrage très différent de celui de M. de Condillac, et vous y approfondirez beaucoup plus la mécanique de notre être» ${ }^{6}$.

Au philosophe français, Bonnet empruntera l'idée d'animer d'abord sa statue par l'odorat, «le sens le moins composé», et non par la vue, comme il l'avait imaginé.

L'Essai de Psychologie nous conduit par degré des premières sensations aux facultés intellectuelles et morales spécifiquement humaines.

Immédiatement après la conception, l'âme est encore passive, «mue plus qu'elle ne meut». Chez le fœtus elle ne connaît pas l'empire qu'elle a sur le corps. Ce n'est qu'à la naissance que sa force motrice commence à se déployer, qu'elle apprend peu à peu à régner sur le corps. «La scène auparavant vide se remplit.» Expression du pouvoir de l'âme, la volonté ne s'exerce qu'après avoir êté nourrie par la sensation. Elle est d'autant plus efficace que les sensations sont plus vives et les perceptions plus distinctes. Au gré des circonstances qui les font naître, les idées se lient progressivement entre elles; elles dépendent des mouvements qui se font dans certaines parties du 
cerveau. L'âme peut les rappeler comme elle peut rappeler l'image d'un objet: en excitant les mêmes fibres. Ainsi s'expliquent la mémoire, la représentation, l'imagination. L'enchaînement des idées conduit à l'universalisation, à la généralisation, et de proche en proche aux notions les plus abstraites: étendue, mouvement, temps, unité, loi, causalité. Avec l'apparition du langage, «qui perfectionne toutes les facultés...», qui accroît indéfiniment la richesse des associations, qui «[multiplie les idées en multipliant] les mouvements de l'organe de la pensée», l'âme va désormais s'affranchir des objets et de leurs images pour ne combiner que les signes qui les représentent. L'être sentant devient un être pensant: il accède à la raison.

Après cette première partie, qui traite «de la diversité des idées et de leur rappel dans l'hypothèse d'un mécanisme organique soumis à la force motrice de l'âme» (MA, p. 171), l'Essai de Psychologie se poursuit par des considérations sur l'habitude et sur l'éducation. Quant à l'Essai analytique, il approfondit et parfois corrige ces premiers développements, et propose aussi une théorie de l'association des idées dont Bonnet tire de nombreuses conséquences pour la morale.

Tous les thèmes abordés dans l'Essai de Psychologie et dans l'Essai analytique s'articulent autour de la théorie des fibres, qui retiendra maintenant notre attention. Bonnet y pose une question dont l'anatomie et la physiologie ne cesseront de débattre, celle de la spécificité des éléments nerveux qui assurent les fonctions sensorielles et cognitives.

«Si toutes nos idées, même les plus spirituelles, dépendent originairement des mouvements qui se font dans le cerveau, il y a lieu de se demander si chaque idée a sa fibre particulière destinée à la produire, ou si la même fibre mue différemment produit différentes idées?» (EP, p. 51).

La question concerne aussi bien les idées abstraites que les idées sensibles. L'idée de rose et l'idée d'œillet, pour prendre l'exemple qui ouvre l'Essai analytique, sont-elles tributaires des mêmes fibres?

Les nerfs ont tous leur origine dans le cerveau et sont en apparence identiques. Comment peuvent-ils alors transmettre à l'âme toute la gamme des impressions que provoque en nous l'infinie diversité des objets? Certes un examen attentif révèle entre les nerfs des différences qui permettent de les classer en plusieurs genres, comme peuvent l'être les sensations et les perceptions. Cela suffit-il à rendre compte de la variété des sensations et des 
perceptions? L'hypothèse de la spécificité des nerfs (ou des fibres) est-elle d'ailleurs nécessaire?

Bonnet cherchera la réponse dans l'examen de la mécanique des idées sensibles.

Le toucher, le goût et l'odorat ne nous offrent rien qui exige que chaque sensation ait sa fibre particulière. Aussi n'est-il pas nécessaire de prêter aux fibres de ces différents appareils sensoriels une grande diversité morphologique: l'organisation fonctionnelle suffit à expliquer la variété des sensations. Voici comment Bonnet se représente l'effet des sels sur les fibres du goût:

«Les uns les picotent: les uns les rongent: les autres les brûlent: d'autres les crêpent: d'autres les contractent: d'autres les distendent: d'autres les secouent...» (EP, p. 59).

Dans le cas des organes de l'ouie et de la vue, Bonnet admet une certaine spécificité. Le fonctionnement des fibres de l'ouie est conçu par analogie avec les cordes d'un instrument, qui produisent un ton en fonction de leur longueur et de leur tension. Chaque fibre de l'ouie ne transmet qu'un seul des sept tons fondamentaux, de même chaque fibre de la rétine une seule des sept couleurs du spectre.

Dans l'Essai analytique, Bonnet, modifiant quelque peu sa conception de la «diversité spécifique» des fibres, sera conduit à admettre

«... qu'il est dans chaque sens des fibres appropriées aux diverses espèces de sensations que le sens peut exciter dans l'âme» (EA, 85).

Que la fibre soit ou non spécifique, la qualité et l'intensité de la sensation dépendent toujours du mouvement que lui communique l'objet ou les corpuscules qui en émanent.

«A un certain degré d'intensité dans le mouvement des fibres répond un certain degré de vivacité dans la sensation» (EP, p.59).

On remarquera combien cette formulation est proche de la loi que Fechner établira environ un siècle plus tard, en jetant les fondements de la psychophysique, mais on observera aussi tout ce qui sépare les idées de Bonnet des conceptions qu'imposera le $\mathrm{XIX}^{\mathrm{e}}$ siècle. S'il paraît annoncer à certains égards la théorie de l'énergie spécifique des nerfs ${ }^{7}$, Bonnet ne conçoit pourtant aucune solution de continuité entre l'objet qui impose son mouvement et les fibres qui le captent. L'objet se retrouve dans la fibre tel qu'en luimême, sans distorsion ni déformation.

Une autre différence majeure sépare les conceptions psycho-physiologiques de Bonnet, qui appartiennent à une période que l'on peut qualifier de 
«pré-corticale», des conceptions modernes, érigées sur l'apport anatomophysiologique du XIX ${ }^{\mathrm{e}}$ siècle. Alors que chez Bonnet la fibre assume seule la spécificité anatomo-fonctionnelle, elle se trouve réduite, dans la physiologie moderne, au rôle de simple «conducteur» (nerfs et faisceaux de substance blanche). Seules la singularisent ses connexions et sa topologie, les agents de sa spécificité étant repoussés aux extrémités: périphérie (dans le récepteur) d'une part et centre (cortex) d'autre part. L'anatomie, à l'époque de Bonnet, n'avait pas encore conquis le cortex, et si l'on veut voir en lui un «prélocalisationniste», il faut dissiper l'équivoque: il «localise» en spécifiant à l'extrême les opérations sensorielles et intellectuelles dont notre âme est capable, mais au sein d'un système dont les éléments entretiennent des relations de juxtaposition, non de hiérarchie.

Bonnet emprunte sa conception anatomique de la fibre à Haller ${ }^{8}$ : c'est un élément creux, constitutif du nerf comme du cerveau, dans lequel circulent les esprits animaux. Nous ignorons, convient Bonnet, la nature de ces esprits, qu'il compare à «un fluide dont la subtilité et la mobilité approchent celles de la lumière», ou encore à un «fluide électrique» (EA, 31). Nous n'admettons leur existence que par la voie du raisonnement, car ils sont «hors de la portée de nos sens et de nos instruments».

Ce qui appartient en propre à Bonnet, ce n'est donc pas une conception «anatomo-physiologique» de la fibre, mais l'idée d'en faire le principe explicatif non seulement de la sensation, mais encore de la vie intellectuelle et mentale dans son ensemble.

Les fibres qui répondent à un objet donné peuvent être activées en son absence; ainsi évoquons-nous ce même objet par l'imagination, par la mémoire. L'habitude peut modifier la fibre, et les préjugés ne sont si tenaces que parce qu'ils sont soumis au fonctionnement des fibres! Aussi bien une fibre qui a été mue une première fois n'est-elle plus dans le même état qu'une fibre vierge. Les fibres subissent des modifications plus ou moins durables, que Bonnet se refuse à caractériser, car il ne trouve aucun fait de nature à l'éclairer sur ce point. Toutefois, étant de très petits organes «... un composé de molécules ou de parties élémentaires dont la forme ou l'arrangement déterminent l'espèce ou le jeu...» (EA, 61), leurs parties constituantes peuvent entrer dans de nouveaux rapports, qui sont les bases physiques de la mémoire.

«En vertu de l'union des deux substances, il ne saurait rien se passer dans l'âme, qui n'ait dans le corps quelque chose qui lui corresponde. C'est cette chose que j'ai toujours cherchée...», 
écrit Bonnet dans l'Analyse abrégée (PP, p. 13). En admettant un parallélisme strict entre les événements psychiques et les événements physiques, et en abordant ces derniers selon le paradigme alors dominant de la mécanique, Bonnet contribue à ouvrir, à la suite de Descartes et avec de nombreux autres savants, un vaste champ d'investigation qui donnera naissance à la psycho-physiologie. Cependant le jeu des éléments nerveux, la mécanique des fibres ne sont pas directement accessibles à l'analyse; il ne peuvent qu'être extrapolés de l'étude des sensations, de la mémoire, de l'imagination, de la pensée, de la volonté, bref du fonctionnement de la vie sensorielle, affective et psychique. Bonnet est contraint d'aller du psychologique au nerveux, d'attribuer au substrat matériel les catégories et les modalités fonctionnelles que l'analyse de la vie sensorielle, intellectuelle et morale tout à la fois lui révèle et lui impose.

Partant des qualités subjectives de la sensation (acuité et diversité de nos sensations, pouvoir discriminatif de nos sens, etc.), de l'organisation de nos facultés que l'introspection nous fait connaître (association d'idées, vivacité des rêves, jeu de la mémoire et de l'imagination, etc.), mais également des objets et des mouvements qui les animent, il en déduit les événements matériels qui se déroulent au sein des fibres, dans les nerfs et le cerveau. Quand il affirme à maintes reprises partir du physique de notre être, c'est d'un postulat qu'il s'agit, non d'une démarche expérimentale. Certes, il obéit scrupuleusement à l'injonction de l'Essai de Psychologie, qu'il «... faut se rendre attentif à ce qui se passe dans l'organe, à la préférence de l'objet» (EP, p.79). Cette déclaration a son importance épistémologique, mais elle annonce un programme plus qu'elle ne témoigne d'une réalisation. L'influence déterminante des éléments physiques sur les processus sensoriels et psychologiques est affirmée, non démontrée.

La volonté de localiser l'union de l'âme et du corps en un point matériellement repérable s'est exprimée au cours des siècles, et surtout depuis Descartes, par de nombreuses suggestions, au gré des découvertes anatomiques et des observations cliniques.

Bonnet se préoccupe peu de localiser anatomiquement le siège de l'âme.

«La découverte de l'origine des nerfs a conduit à placer l'âme dans le cerveau. Mais comme il n'y a que les corps qui aient une relation proprement dite avec le lieu, nous ne dirons pas que l'âme occupe un lieu dans le cerveau, nous dirons que l'âme est présente au cerveau, et par le cerveau à son corps d'une manière que nous ne pouvons définir» (EA, 27).

A un certain état du corps répond constamment un certain état de l'âme, mais «le mystère de l'union [reste] impénétrable au philosophe». Bonnet y 
voit un des abîmes les plus profonds qui soient dans le pays des connaissances humaines.

Pourtant, un peu plus loin dans ce même Essai analytique, il admet

«... qu'il est quelque part dans le cerveau une partie que je nomme le siège de l'âme, et que je regarde comme l'instrument immédiat du sentiment, de la pensée, et de l'action» (EA, 29).

Insensiblement, en quelques lignes, se dessinent deux conceptions irréductibles; elles expriment les deux influences majeures entre lesquelles hésite la pensée de Bonnet, le dualisme cartésien et le sensualisme.

«Le siège de l'âme serait ainsi un centre où tous les nerfs iraient rayonner» (EA, 30),

écrit encore Bonnet, car cet organe si prodigieusement composé, cette «neurologie en miniature», doit communiquer avec tous les sens et permettre d'agir sur toutes les parties du corps. Une nouvelle façon de poser le problème des localisations anatomiques s'amorce ici. Il ne s'agira plus, désormais, de situer le siège de l'âme, mais de localiser des facultés dans les diverses régions du cerveau. Bonnet compare cet organe tantôt à un clavecin dont l'âme serait le musicien, tantôt à une horloge, et y suppose l'existence de zones motrices et sensorielles. Sans doute n'est-il pas le premier à développer ces métaphores «localisatrices», mais la prééminence qu'il accorde aux fibres et aux organes des sens contribue à la naissance d'une nouvelle anatomie fonctionnelle du cerveau : en suivant les voies sensorielles, on découvrira de nouveaux principes d'organisation cérébrale.

Bonnet se défend de l'accusation de matérialisme (EA, 75), et certes on ne saurait douter de son attachement au christianisme, longuement exprimé dans la préface à l'Essai de psychologie. A maintes reprises dans son œuvre il proclame l'immatérialité de l'âme, qu'il cherche à étayer sur diverses preuves. Il se plaît même à imaginer le sort de l'âme humaine logée dans une huître: elle y serait atrocement malheureuse, limitée à un appareil nerveux et sensoriel aussi pauvre. Il propose néanmoins une explication organique, matérielle, de la vie psychique:

«Si l'âme d'un Huron eût pu hériter du cerveau de Montesquieu, Montesquieu créerait encore» $(\mathrm{EA}, 771)$.

Le cerveau devient le dépositaire des facultés qui appartenaient traditionnellement à l'âme, mais, ajoute Bonnet, 
«en transportant au corps des choses que l'on attribue communément à l'âme, je ne dégrade que l'âme, et je ne dégrade point notre être» (EA, 95).

L'âme n'est pas en soi supérieure au corps matériel, c'est simplement une hypothèse dont on ne peut se passer. Comment l'organisation matérielle pourrait-elle rendre compte de la simplicité du moi, de son caractère unique et indivisible? (EA, préface, et PP, p. 48). Irréductible à la notion d'étendue, l'âme dans ses manifestations les plus hautes, dans la conscience et le sentiment de l'unité du moi, ne saurait être corporelle.

«Si quelqu'un démontrait jamais que l'âme est matérielle, loin de s'en alarmer, il faudrait admirer la PUISSANCE qui aurait donné à la matière la capacité de penser» (PP, p. 50).

Par cette dernière parade, Bonnet se préserve du matérialisme tant redouté. Ses convictions chrétiennes n'ont cependant jamais fait obstacle à l'audace de ses vues, elles n'ont pas troublé la tranquille assurance qui l'a conduit à engager l'étude de nos facultés - celles que l'homme partage avec l'animal comme celles qui lui sont propres - dans une voie nouvelle qui prenait en compte les conditions matérielles de leur possibilité.

\section{Notes}

1 Savioz Raymond, Mémoires Autobiographiques de Charles Bonnet de Genève, Paris, Vrin, 1948, p. 167. Les références ultérieures à cet ouvrage seront indiquées par l'abréviation MA.

2 Préface à l'Essai Analytique. L'influence des Mémoires pour servir à l'Histoire des Insectes de Réaumur fut précoce et déterminante pour Bonnet. Il lui doit des préceptes méthodologiques dont il fera partout usage.

3 L'Essai de Psychologie fut publié en 1754 à Leyde sans nom d'auteur; il porte la date de 1755 et indique un lieu fictif, Londres.

4 Edité à Copenhague en 1760.

5 Edité à Genève, chez Jean-Marie Bruyset, en 1770.

6 Le pasteur Laget, qui fut son précepteur. L'anecdote est rapportée dans les Mémoires autobiographiques, p. 176-177.

7 La théorie de l'énergie spécifique des nerfs a été formulée par Johannes Müller (1801-1858). Dans cette théorie, le «stimulus» est conceptualisé sous la forme d'une énergie (mécanique, thermique, etc.) et l'idée commence à se faire jour qu'il faut extraire de l'objet des caractéristiques pertinentes. Chez Bonnet, l'infinie variété des objets empiriques exige un nombre égal d'éléments spécifiés (fibres) capables de les percevoir.

8 Dans le premier chapitre de ses Eléments de physiologie, Haller expose sa conception de la fibre. «Les parties solides, tant des animaux que des végétaux, ont cela de commun dans 
leur structure, que les plus petits éléments de ces parties, découverts à l'aide du microscope, sont ou fibre ou masse inorganisée.» Les fibres que la dissection nous révèle sont ellesmêmes constituées d'unités élémentaires, appelées fibres simples, et que «la raison, plutôt que les sens» nous fait apercevoir. Les fibres composées se présentent sous deux formes différentes: fibres linéaires d'une part, composées de fibrilles élémentaires, toutes cylindriques et parallèles (os, tendons, ligaments, muscles), tissu cellulaire d'autre part, plus large que long, composé de fibrilles et de petites lames, laissant entre elles des espaces (vaisseaux, membranes, tissus de soutien, etc.). Les fibres nerveuses sont bien sûr linéaires. Haller considère qu'elles sont creuses et exercent leurs fonctions au moyen du liquide qu'elles renferment, et non par leur élasticité. Il désespère pourtant de jamais découvrir comment les nerfs mettent les muscles en mouvement!

\section{Summary}

\section{Soul and sensation according to Charles Bonnet (1720-1793)}

When Charles Bonnet, the many-sided naturalist, was forced by eye troubles to abandon observation and experimentation, he took to psychology and philosophy, concentrating his thought on two main topics: the origin of our ideas and the organisation of our mental faculties. In Bonnet's view, the nerve fibre is the anatomical and physiological element on which not only sensation but the entire mental life depends.

\section{Zusammenfassung}

\section{Seele und Sinneswahrnehmung nach Charles Bonnet (1720-1793)}

Als der vielseitige Naturforscher Bonnet wegen eines Augenleidens auf die Naturbeobachtung verzichten mußte, wandte er sich der Psychologie und der Philosophie zu. Seine Überlegungen galten zwei Hauptthemen: dem Ursprung unserer Ideen und der Organisation unserer psychischen Fähigkeiten. Dabei wird die Nervenfaser zum anatomisch-physiologischen Grundelement nicht nur für die Sinnesempfindung, sondern für das gesamte geistige Leben.

Patrick Baud

12, rue Adrien Lachenal

1207 Genève 